\title{
Search for Novel Origins of Cosmic-ray Antiprotons and Antimatter with BESS-Polar Flight over Antarctica.
}

\section{Koji YOSHIMURA*}

High Energy Accelerator Research Organization (KEK), Tsukuba, Ibaraki 305-0801 Japan E-mail: koji.yoshimura@kek.jp

S. Haino, M. Hasegawa, A. Horikoshi, T. Kumazawa, Y. Makida, S. Matsuda, K. Matsumoto, M. Nozaki, J. Suzuki, K. Tanaka, A.Yamamoto

High Energy Accelerator Research Organization (KEK), Tsukuba, Ibaraki 305-0801, Japan

\section{T. Hams, J.W. Mitchell, A.A. Moiseev, R.E. Streitmatter, M. Sasaki}

NASA/Goddard Space Flight Center, Greenbelt, MD 20771 USA

\section{K. Abe, Itazaki, Y. Matsukawa, Y. Shikaze, R. Orito, Y.Takasugi,K. Takeuchi \\ Kobe University, Kobe, Hyogo, 657-8501 Japan}

\section{H. Fuke, T. Yamagami, T. Yoshida}

Institute of Space and Astronautical Science (ISAS/JAXA), Sagamihara, Kanagawa, 229-8510 Japan

K.C. Kim, M.H. Lee, Z. Myers, E.S.Seo

IPST, University of Maryland, College Park, MD 20742 USA

\section{J. Nishimura, K. Sakai, R. Shinoda}

The University of Tokyo, Bunkyo, Tokyo, 113-0033 Japan

\section{J.F. Ormes, N. Thakur}

Denver University, Denver, CO 80208 USA

The primary aims of the BESS-Polar program are precise measurements of the low-energy antiproton spectrum and search for cosmologically significant antimatter, which would provide new clues to understand the early Universe. The second flight (BESS-Polar II) over Antarctica was successfully carried out in Dec 2007 - Jan 2008. We performed 24.5days scientific observation just at the solar minimum. The payload worked well during the flight and 4.7 billion cosmic-ray events were collected. Based on the BESS-Polar II data, we present recent preliminary results of cosmic-ray antiproton measurements and sensitive search for antimatter.

35th International Conference of High Energy Physics - ICHEP2010,

July 22-28, 2010

Paris France

\footnotetext{
* Speaker.
} 


\section{INTRODUCTION}

The objectives of the BESS-Polar experiment are definitive measurements of various lowenergy cosmic-ray phenomena with high statistics; particularly providing a firm answer on possible primary component of cosmic-ray antiprotons, searching for antinuclei down to an unprecedented level, and studying the origin and propagation of other galactic cosmic-ray species. After its first successful flight in 2004 (BESS-Polar I), the second flight of BESS-Polar (BESS-Polar II) has been prepared to be carried out at the solar minimum with an improved instrument and capability for an extended long-duration flight.

BESS-Polar II payload was successfully launched from the Williams Field on December 23, 2007. The payload flew with one and 3/4 circumnavigation over Antarctica in 29.5 days. Science observation was successfully performed during 24.5 day science observation. Detailed information about BESS-Polar II spectrometer and flights are described in the reference[1].

\section{MEASUREMENT OF ANTIPROTON}

Among 4.7 billion cosmic-ray events recorded onboard, antiproton candidates were extracted according to particle identification information. Figure 1 shows $1 / \beta$ vs. rigidity for events which survive all selection with $\mathrm{dE} / \mathrm{dx}$ of TOF, MTOF, and JET and Aerogel veto. In the figure, band structure is clearly seen, each of which corresponds to mass of the particle species, e.g. proton, deuteron, triton and so on. We can identify nearly 8000 antiprotons within the band just at the opposite position as proton.

Based on identified antiproton and proton samples, we obtained antiproton/proton ratio[2]. We only used last 1/4 data for this preliminary analysis, conservatively, since performance of tracking detector as well as trigger condition were stable in this time window. Figure 2 shows preliminary antiproton/proton flux ratio using 1/4 statistics of BESS-Polar II. Data are compared with previous BESS data and PAMELA data reported recently [3].

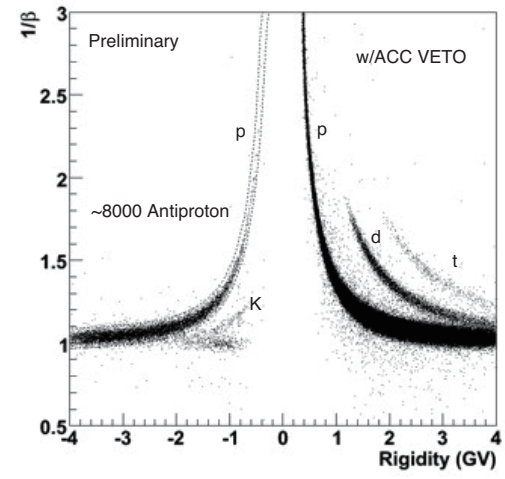

Figure 1: $1 / \beta$ vs Rigidity for the event samples with $A C C$ veto.

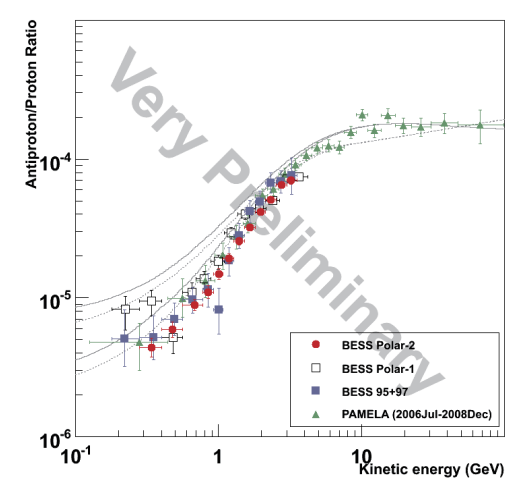

Figure 2: Antiproton/Proton ratio for last 1/4 period of BESS-Polar II. Analysis in the lowest energy bin is still in progress by using MTOF. 


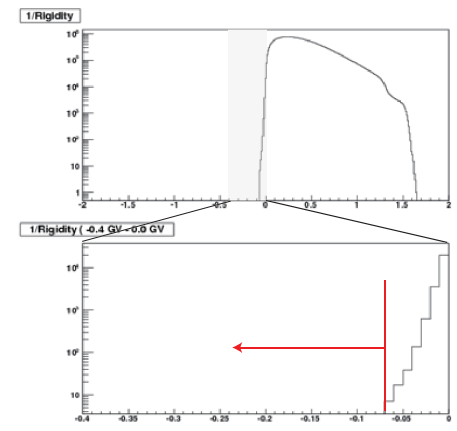

Figure 3: 1/Rigidity distribution for all identified helium/antihelium samples

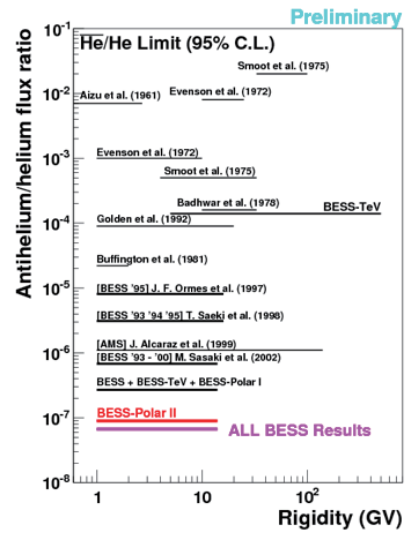

Figure 4: New preliminary upper limit on antihelium/helium ratio.

\section{SEARCH FOR ANTIHELIUM}

To select charge $\mathrm{z}=2$ events(helium/antihelium), track quality cut and $\mathrm{dE} / \mathrm{dx}$ cut were applied after single track selection. Separation of helium and antihelium was performed by using curvature of the track. Figure 3 shows inverse rigidity distribution for the event samples which are identified as helium and antihelium. We can see spill over from positive rigidity side to negative due to finite resolution in rigidity measurement. No antihelium candidate was not found in the rigidity -14 GV $<\mathrm{R}<-1 \mathrm{GV}$ among $4 \times 10^{7}$ helium events.

Based on the number of the helium events, we preliminarily placed upper limit on antihelium/helium ratio [4] in the rigidity range of $-14 \mathrm{GV}<\mathrm{R}<-1 \mathrm{GV}$ as follows (Fig 44) :

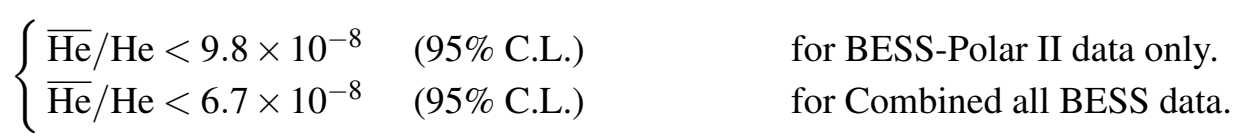

\section{SUMMARY}

Antiproton/proton ratio except lowest energy region was very preliminary obtained, based on $1 / 4$ statistic of BESS-Polar II data. Analysis with full statistics and low energy region is now in progress. We searched for antihelium in all of BESS-Polar II data. No antihelium candidate was observed and we set a preliminary upper limit on antihelium/helium ratio.

\section{References}

[1] J.W.Mitchell et al., "The balloon-borne experiment with a superconducting spectrometer (BESS) program"; K. Yoshimura et al., "Detector performances of the BESS-Polar II instrument during the second long-duration balloon flight over Antarctica",38th COSPAR, July 2010 Bremen Germany.

[2] K. Sakai et al., "Measurement of comic-ray antiproton spectrum at solar minimum with the BESS-Polar II flight in Antarctica", 38th COSPAR, July 2010 Bremen Germany.

[3] O. Adriani et al.,"PAMELAResults on the Cosmic-Ray Antiproton Flux from $60 \mathrm{MeV}$ to $180 \mathrm{GeV}$ in Kinetic Energy", Phys. Rev. Lett. 105, 121101 (2010).

[4] M. Sasaki et al., "Search for Antihelium with BESS-Polar II", 38th COSPAR, July 2010 Bremen Germany. 